\title{
Fasting enhances mucosal antigen specific B cell responses in rheumatoid arthritis
}

\author{
C Trollmo, M Verdrengh, A Tarkowski
}

\begin{abstract}
Objective-To evaluate the influence of fasting on the antigen specific immune responsiveness in patients with rheumatoid arthritis and healthy volunteers.

Methods-Seven rheumatoid arthritis patients and 17 healthy volunteers were immunised perorally or parenterally with influenza virus vaccine after a three to six day long period of total energy deprivation (water fast). The subsequent antigen specific antibody mediated immune response was recorded in the blood at the single cell level by the ELISPOT method. Results-Short term starvation induced an enhanced antigen specific mucosa derived $B$ lymphocyte response in rheumatoid arthritis patients and healthy volunteers. In contrast, the systemic $B$ cell responses were not significantly altered by a total energy deprivation.

Conclusions-Short term starvation increases the mucosa derived $B$ cell responsiveness, while systemic responsiveness is largely unaffected. The similar pattern of response in rheumatoid arthritis patients and healthy volunteers indicates that fasting alters the mucosal immune response independently of medical treatment.
\end{abstract}

(Ann Rheum Dis 1997;56:130-134)

The question whether food causes or cures arthritis has been posed for many decades, but nutritional management of arthritis is still considered an experimental procedure. ${ }^{1}$ In contrast, it is well established that total fasting gives rise to a temporary improvement in inflammatory variables, pain, and stiffness in patients with rheumatoid arthritis. ${ }^{2}$ However, the mechanisms for the anti-inflammatory effects of this acute total energy deprivation remain to be elucidated. ${ }^{3}$ The modulating effect of fasting on disease activity on rheumatoid arthritis strengthens the idea of an involvement of the gut in the pathogenesis of the disease. ${ }^{4}$ Gut permeability and intestinal flora have been shown to be abnormal in rheumatoid arthritis patients, ${ }^{56}$ while the intestinal humoral immune response to protein antigens is intact. ${ }^{7}$

The objective of this study was to evaluate whether a total fast influences the immune response to a specific antigen given either parenterally or perorally to patients with rheumatoid arthritis and to healthy volunteers.

\section{Methods}

SUBJECTS AND VACCINE

Table 1 gives a summary of the experimental procedures and clinical features of the participants in this study. The patients with rheumatoid arthritis, fulfilling the ARA criteria for the diagnosis, ${ }^{8}$ continued their medication during the whole study period. Since the participants in the study have previously been naturally exposed to the influenza virus, we considered that all $\mathrm{B}$ cell responses to the influenza vaccine (kindly donated by Merieux, Lyon, France) are of a secondary nature.

In the first set of experiments six patients with rheumatoid arthritis and 12 healthy volunteers were immunised perorally with the influenza virus vaccine after a three or six day fast on water. Five millilitres of the influenza virus vaccine was dissolved in tap water containing $1 \mathrm{~g}$ of bicarbonate immediately before intake. To compensate for the big interindividual variations in the immune response to influenza vaccine, matched controls were provided by immunizing the same individuals in a non-fasting state a second time within a month with the same vaccine. Thus all the patients served as their own controls. The non-fasting immunisations were performed after an overnight fast.

For control purposes, four healthy non-fasting volunteers were immunised twice perorally within a month to analyse the potential booster effect of two peroral influenza immunisations.

In the second set of experiments, one patient with seronegative rheumatoid arthritis and four healthy volunteers were immunised parenterally after a three or six day water fast. Control immunisation followed within a month. According to the manufacturer's recommendations, $0.5 \mathrm{ml}$ of the vaccine was given subcutaneously. To analyse a potential booster effect of two parenteral influenza immunisations, three healthy volunteers were immunised twice within a short time period. The study was approved by the ethics committee of the University of Göteborg.

ISOLATION OF LYMPHOCYTES FROM PERIPHERAL BLOOD

Blood was collected before the fast, after the fast connected with the immunisation, and seven days later, since $B$ cell responses at the single cell level peak at day 7 after both systemic and peroral immunisations. ${ }^{79}$ Peripheral blood mononuclear cells (lymphocytes) were isolated by centrifugation on a FicollHypaque density gradient (Sigma Chemicals, St Louis, MO, USA), washed, and resuspended in Iscoves medium (Gibco, Glasgow, 
Table 1 Summary of experimental procedures and clinical features of subjects included in this study

\begin{tabular}{|c|c|c|c|c|c|c|c|c|c|}
\hline \multirow[b]{2}{*}{$\begin{array}{l}\text { Route of } \\
\text { immunisation }\end{array}$} & \multirow[b]{2}{*}{ Diagnosis } & \multirow[b]{2}{*}{$\begin{array}{l}\text { No of } \\
\text { fasting } \\
\text { days }\end{array}$} & \multirow[b]{2}{*}{$\begin{array}{l}\text { No of } \\
\text { subjects }\end{array}$} & \multirow[b]{2}{*}{$\begin{array}{l}\text { Age (years, } \\
\text { mean (SD)) }\end{array}$} & \multirow[b]{2}{*}{$\begin{array}{l}\text { Sex } \\
(M / F)\end{array}$} & \multirow[b]{2}{*}{$\begin{array}{l}\text { Disease duration } \\
\text { (years, mean } \\
(S D))\end{array}$} & \multicolumn{3}{|l|}{ Treatment } \\
\hline & & & & & & & Corticosteroids & $D M A R D$ & $\begin{array}{l}\text { Cytotoxic } \\
\text { drugs }\end{array}$ \\
\hline \multirow[t]{5}{*}{ Peroral } & Seropositive RA & 6 & 5 & $49(7)$ & $0 / 5$ & $15(6)$ & $80 \%$ & $60 \%$ & $20 \%$ \\
\hline & Seronegative polyarthritis & 3 & 1 & 60 & $0 / 1$ & 1 & None & None & None \\
\hline & Controls & 6 & 5 & $36(7)$ & $3 / 2$ & - & - & - & - \\
\hline & Controls & 3 & 7 & $40(8)$ & $3 / 4$ & - & - & - & - \\
\hline & Controls & None & 4 & $38(10)$ & $1 / 3$ & - & - & - & - \\
\hline \multirow[t]{3}{*}{ Parenteral } & Seronegative polyarthritis & 3 & 1 & 61 & $0 / 1$ & 2 & None & None & None \\
\hline & Controls & 3 & 5 & $40(10)$ & $2 / 3$ & - & - & - & - \\
\hline & Controls & None & 3 & $42(12)$ & $1 / 2$ & - & - & - & - \\
\hline
\end{tabular}

DMARD, disease modifying antirheumatic drugs.

UK) supplemented with $5 \%$ fetal calf serum (FCS) (Gibco). The lymphocytes were used immediately after isolation for the enzyme linked immunospot assay (ELISPOT) as previously described. ${ }^{10} 11$

REAGENTS

Affinity purified goat anti-human IgG, heavy and light chain specific (Jackson Laboratories, PA, USA), diluted in phosphate buffered saline (PBS) to a concentration of $5 \mu \mathrm{g} \mathrm{ml}^{-1}$, and influenza vaccine, diluted 1:20 in PBS, were used as coating reagents in the ELISPOT assay. ${ }^{10}$ The coating concentrations were previously optimised by checkerboard titration. Affinity purified, biotinylated $\mathrm{F}\left(\mathrm{ab}^{\prime}\right)_{2}$ fragments of goat anti-human IgG, IgA, and IgM antibodies (Tago, Burlingame, CA, USA) were used at the developing stage. Avidin-alkaline phosphatase (ALP) was purchased from Sigma.

ELISPOT ASSAY FOR ENUMERATION OF IMMUNOGLOBULIN AND SPECIFIC ANTIBODY SECRETING CELLS

The ELISPOT assay was used to determine the numbers of immunoglobulin secreting cells of a given isotype and of antigen specific antibody secreting cells. Briefly, wells were coated (see "Reagents") overnight at $4^{\circ} \mathrm{C}$ and blocked with $5 \%$ PBS-FCS for one hour. One hundred microlitres of mononuclear cell suspension were added into each well and incubated for 3.5 hours at $37^{\circ} \mathrm{C}$. After washing, biotinylated goat anti-human $\operatorname{IgA}, \operatorname{IgG}$, and $\operatorname{IgM}$ antibodies, diluted 1:750 in PBS containing $0.05 \%$ Tween 20 , were applied followed by incubation with $0.5 \mathrm{mg} \mathrm{ml}^{-1}$ of avidin-ALP in PBS-Tween. After addition of the phosphatase substrate solution (5-bromo-4-chloro-3indolyl phosphate) (Sigma) spots were enumerated. Each spot represents one immunoglobulin secreting cell (SFC). All the assays were done in duplicate and at cell concentrations of $1 \times 10^{5}, 5 \times 10^{5}, 1 \times 10^{6}$, and $1 \times 10^{7} \mathrm{ml}^{-1}$.

\section{STATISTICAL ANALYSIS}

Statistical analysis regarding differences between groups was carried out by the MannWhitney test. Differences within groups were analysed by the Wilcoxon signed rank test.

\section{Results}

INFLUENCE OF FASTING ON THE MUCOSAL B CELL RESPONSE

To assess the effect of a total energy deprivation on the mucosa derived influenzaspecific immune response, healthy volunteers and patients with rheumatoid arthritis were perorally immunised with an influenza vaccine and the subsequent antibody production was recorded in the blood at the single cell level.

Lymphocytes from six perorally immunised rheumatoid arthritis patients and 12 healthy controls were analysed before the fast, after the three or six day long fast connected with the influenza immunisation, and seven days later to assess the frequency of antigen specific and
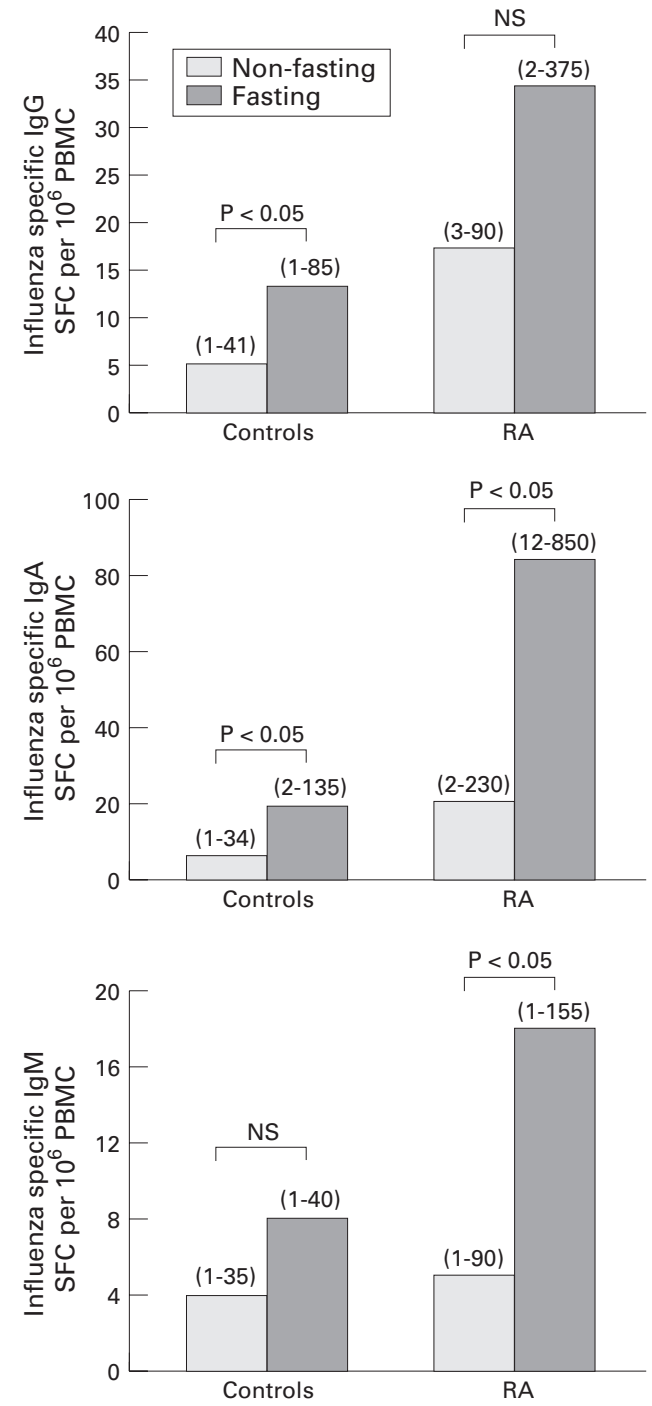

Influenza specific immunoglobulin secreting cells (SFC) of IgG, IgA, and IgM classes in fasting and non-fasting rheumatoid arthritis patients $(n=6)$ and healthy volunteers $(n=12)$. The results are presented as geometric means (range). 
Table 2 Numbers of total immunoglobulin secreting cells (SFC IgG+IgA+IgM) in peripheral blood in fasting subjects at the different time points indicated. Results are expressed as geometirc means (range) of SFC per $10^{6}$ lymphocytes

\begin{tabular}{|c|c|c|c|c|}
\hline \multirow[b]{2}{*}{ Day of analysis } & \multicolumn{2}{|c|}{ Peroral immunisation } & \multicolumn{2}{|c|}{ Parenteral immunisation } \\
\hline & Controls $(n=12)$ & $R A(n=6)$ & Controls $(n=4)$ & $R A(n=1)$ \\
\hline Before fasting & $683(310-1700)$ & $1826(1030-3240)$ & $749(570-1070)$ & 2890 \\
\hline Directly after fasting & $699(315-2670)$ & $1135(610-1600)$ & $841(520-1660)$ & 2840 \\
\hline Seven days after fasting & $899(340-3230)$ & $1467(800-2330)$ & $1596(1030-3210)$ & 1720 \\
\hline Before control immunisation & $476(130-970)$ & $984(430-1690)$ & $536(300-990)$ & 830 \\
\hline Seven days after control immunisation & $740(145-3660)$ & $1219(800-2490)$ & $428(200-900)$ & 1380 \\
\hline
\end{tabular}

RA, rheumatoid arthritis.

total immunoglobulin spot forming cells. Lymphocytes were also analysed before and seven days after the immunisation without prior fasting, as indicated above. Before the immunisations no antigen specific SFC were detected. However, seven days later antigen specific SFC were found in the majority of the immunised subjects, in both fasting- and non-fastingindividuals (figure). Antibody responses after short and long term fasting did not show any significant differences and thus were pooled.

In both fasting groups the geometric means of influenza specific SFC of IgG, IgA, and IgM isotypes were higher seven days after the peroral immunisation compared with the corresponding time in the non-fasting controls (figure). Statistical calculations showed that the increase of influenza specific IgA SFC between fasting and non-fasting immunisations was significant in both rheumatoid arthritis patients and healthy volunteers $(\mathrm{P}=$ 0.03 ), as were the increases in $\operatorname{Ig} M$ influenza specific SFC in rheumatoid arthritis patients $(P=0.04)$ and IgG influenza specific SFC in the control subjects $(P=0.04)$. Increases of antigen specific antibody responses, measured as SCF, in the non-fasting versus the fasting state were not significantly different between the rheumatoid arthritis patients and the healthy controls for any of the isotypes analysed. Analysis of the isotype distribution of the influenza specific SFC after peroral immunisation showed a similar isotype distribution in the patients and the controls for both fasting and non-fasting immunisations: $30 \%$ of $\operatorname{IgG}$ SFC, $50 \%$ of IgA SFC, and $20 \%$ of IgM SFC.

Total immunoglobulin production was analysed in parallel: before the fast, after the fast connected with the immunisation, and seven days after the immunisation, as well as before and seven days after the control immunisation. The number of immunoglobulin SFC of the three isotypes (IgG, IgA, and $\operatorname{IgM}$ ) did not differ significantly either within groups or between groups with respect to fasting or immunisation procedures (table 2).

The proportion of influenza specific SFC $(\operatorname{IgG}+\operatorname{Ig} \mathrm{A}+\operatorname{Ig} M)$ in relation to the overall immunoglobulin SFC $(\operatorname{IgG}+\operatorname{IgA}+\operatorname{IgM})$ on day 7 after fasting and non-fasting immunisations was compared. After the fast, $10 \%$ of total immunoglobulin SFC was antigen specific as compared to $4 \%$ after the immunisation in the non-fasting subjects.

For control purposes four healthy volunteers were immunised twice perorally one month apart with the influenza vaccine. The numbers of anti-influenza-specific SFC of IgG, IgA, and IgM classes seven days after the first and second immunisation were similar for each individual. The geometric mean values for the two immunisations were 20 and 34 antigen specific SFC, respectively (NS).

INFLUENCE OF FASTING ON THE SYSTEMIC B CELL RESPONSE

To assess the effect of a total fast on the systemic immune responsiveness, healthy volunteers and a patient with seronegative polyarthritis were parenterally immunised with an influenza vaccine after three days of fasting and the subsequent immune response was recorded at the single cell level. Lymphocytes were analysed before the fast, after the fast connected with the influenza immunisation, and seven days later for frequency of antigen specific and total immunoglobulin SFC. Lymphocytes were also analysed as described above before and seven days after the immunisation without prior fasting. Before the immunisations no antigen-specific SFC were detected. However, seven days later antigen specific SFC were found in all immunised subjects, irrespective of the nutritional status. As with the three healthy volunteers immunised twice parenterally without prior fasting, no significant differences with respect to the mean values of antigen specific SFC were seen on day 7 after the fasting and non-fasting immunisations. The control group differed in the same way between the two immunisations.

Parenteral immunisation induced the following isotype distribution in healthy controls: $65 \%$ of IgG SFC, $30 \%$ of IgA SFC, and $5 \%$ of IgM SFC. No major differences with respect to the isotypic distribution were seen between the fasting and non-fasting immunisations.

The total immunoglobulin production was analysed in parallel with the influenza specific SFC before the fast, after the fast connected with the immunisation, and seven days after immunisation, and before and seven days after the control immunisation. The numbers of immunoglobulin SFC of the three isotypes (IgG, IgA, and IgM) did not differ significantly either within groups or between groups with respect to fasting and immunisation procedures (table 2).

The proportions of influenza specific SFC to total immunoglobulin SFC on day 7 after fast and control immunisations were compared. After both immunisations about $20 \%$ of the total number of immunoglobulin producing cells were influenza specific SFC. 


\section{Discussion}

To our knowledge this is the first study to show that three to six days of total energy deprivation significantly increases antigen specific mucosa derived $\mathrm{B}$ lymphocyte response in rheumatoid arthritis patients and healthy volunteers. In contrast, the systemic B cell response to the same immunogen was not significantly affected by a total fast.

Several possibilities could explain the greater antibody response seen after the fasting period.

(1) Antigen uptake through the gut epithelial cells or $M$ cells can be increased during the fasting because of changed cellular permeability. ${ }^{12}$ Despite administration of non-steroidal anti-inflammatory drugs, which alter gut permeability, ${ }^{13}$ the mucosa derived immune response after fasting was similar in the two study groups. Therefore, it is unlikely that medication per se affected the immune responsiveness.

(2) Several days of fasting could give rise to higher local antigenic availability, leading to increased immune responses. However, even the so called non-fasting controls were given the influenza vaccine perorally after an overnight fast, when the small intestine was empty of food products.

(3) An increase in the production of B cell stimulatory, $\mathrm{T}$ cell derived cytokines could also explain the increased $\mathrm{B}$ cell responsiveness after fasting. Our results from in vitro cytokine production in response to influenza vaccine showed no increase in $\mathrm{B}$ cell differentiating cytokines like interleukin (IL)-4, IL-10, and interferon $\gamma$ (results not shown). However, the local activity of intestinal $\mathrm{T}$ cells remains to be elucidated.

(4) During starvation many hormonal changes occur. ${ }^{14}$ Although certain hormones have been shown to affect immunoglobulin production, ${ }^{15}$ we exclude the possibility that they would act only locally and affect mucosal but not systemic B cell responses.

(5) We favour the possibility that more antigen presenting cells are available or activated in the villi of the gut or in the adjacent lymph nodes during the fasting period. In this respect, earlier studies have shown enhanced peripheral blood monocyte activity after 10 days of total food deprivation. ${ }^{16}$

We can easily exclude an effect of a booster immunisation as an explanation for the increased mucosal immune response after fasting since (a) in all but one immunised subject the non-fasting immunisation followed the fasting one, and (b) a similar magnitude of immune response was measured after two peroral immunisations within a short time period in non-fasting controls.

We have recently shown that mucosal deposition of influenza virus vaccine induces an antigen specific immune response in the circulation, the magnitude and isotypic specificity of which are similar in rheumatoid arthritis patients and healthy volunteers. ${ }^{7}$ Our present results show that the stimulatory effect of fasting on the mucosally triggered B lymphocyte response is also similar in the two groups studied.
In the parenterally immunised subjects there was no significant difference in the humoral influenza specific immune response according to whether there was prior fasting or not. This strengthens the notion that the gut associated lymphoid tissue is affected by fasting, while the systemic immune system is not. However, in this respect our results are in contrast to an earlier study which showed an increased antigen specific antibody response to a primary systemic immunisation with flagellin in fasting compared to non-fasting subjects. ${ }^{17}$ The differences between these results might partly depend on the differences between primary ${ }^{17}$ and booster (present study) immunisation procedures. It should also be noted that our systemically immunised group was rather small (four healthy volunteers and one patient).

In summary, although gut homeostasis and medical treatment are different in rheumatoid arthritis patients and healthy volunteers, the pattern with an increased mucosa triggered immune responsiveness after fasting as compared to a non-fasting immunisation was the same in the two groups. Further studies are required to pinpoint the events causing augmentation of the $\mathrm{B}$ cell responses as a result of fasting.

In view of the decreased systemic immune responsiveness in rheumatoid arthritis patients, our results could be used in future enteric vaccination/tolerisation procedures.

Supported by grants from Börje Dahlins foundation, the Göteborg Medical Society, the King Gustav V:s 80 Years Foundation, the Nanna Svartz Foundation, the Swedish Association against Rheumatism, the Swedish Medical Research Council, the A-G Crafoord Foundation, and the University of Göteborg. We thank Dr Monica Ahlmen, Rheumatology Unit, Mölndals Hospital, Mölndal, Sweden, for providing us with some of the patients.

1 Panush RS. Does food cause or cure arthritis? Rheum Dis Clin North Am 1991;17:259-72.

2 Hafström I, Ringertz B, Gyllenhammar H, Palmblad J, Harms-Ringdahl M. Effects of fasting on disease activity, neutrophil function, fatty acid composition, and leukotriene biosynthesis in patients with rheumatoid arthritis. riene biosynthesis in patients with
Arthritis Rheum 1988;31:585-92.

3 Palmblad J, Hafström I, Ringertz B. Antirheumatic effects of fasting. Rheum Dis Clin North Am 1991;17:351-62.

4 Kjeldsen-Kragh J, Haugen M, Borchgrevink CF, Laerum E, Eek M, Mowinkel P, et al. Controlled trial of fasting and one-year vegetarian diet in rheumatoid arthritis. Lancet 1991;338:899-902.

5 Hazenberg MP, Klasen IS, Kool J, Ruseler-van Embden $\mathrm{JGH}$, Severijnen AJ. Are intestinal bacteria involved in the etiology of rheumatoid arthritis? APMIS 1992;100:1-9.

6 Smith MD, Gibson RA, Brooks PM. Abnormal bowel permeability in ankylosing spondylitis and rheumatoid arthritis. J Rheumatol 1985;12:299-305.

7 Trollmo C, Sollerman C, Carlsten H, Tarkowski A. The gut as an inductive site for synovial and extra-articular immune responses in rheumatoid arthritis. Ann Rheum Dis 1994;53:377-82.

8 Arnett FC, Edworthy SM, Bloch DA, McShane DJ, Fries JF, Cooper NS, et al. The American Rheumatism Association 1987 revised criteria for classification of rheumatoid tion 1987 revised criteria for classification of

9 Tarkowski A, Czerkinsky C, Nilsson L-A.. Simultaneous induction of rheumatoid factor- and antigen-specific antibody-secreting cells during the secondary immune response in man. Clin Exp Immunol 1985;61:379-87.

10 Czerkinsky C, Nilsson LÅ, Nygren H, Ouchterlony Ö, Tarkowski A. A solid phase enzyme-linked immunospot (ELISPOT) assay for enumeration of specific antibodysecreting cells. J ImmunolMethods 1983;65:109-21.

11 Trollmo C, Carlsten H, Tarkowski A. Intra-articular immunisation induces strong systemic immune response in humans. Clin Exp Immunol 1990;82:384-9.

12 Sundqvist T, Lindström F, Magnusson K-E, Sköldstam L, Stjernström I, Tagesson C. Influence of fasting on intestinal permeability and disease activity in patients with rheumatoid arthritis. Scand J Rheumatol 1982;11:33-8.

13 Bjarnason I, So A, Levi AJ, Peters TJ, Williams P, Zanelli $\mathrm{GP}$, et al. Intestinal permeability and inflammation in 
rheumatoid arthritis: effects of non-steroidal antiinflammatory drugs. Lancet 1984;ii:1171-3.

14 Beer SF, Bircham PMM, Bloom SR, Clark PM, Hales CN, Hughes CM, et al. The effect of a 72 -h fast on plasma levels of pituitary, adrenal, thyroid, pancreatic and gastrointestinal hormones in healthy men and women. J Endocrinol 1989;120:337-50.

15 Blalock JE. A molecular basis for bidirectional communication between the immune and neuroendocrine systems. Physiol Rev 1989;69:1-32.
16 Wing EJ, Stanko RT, Winkelstein A, Adibi SA. Fastingenhanced immune effector mechanisms in obese subjects. Am J Medicine 1983;75:91-6.

17 Palmblad J, Cantell K, Holm G, Norberg R, Stranders H, Sunblad L. Acute energy deprivation in man:effect on serum immunoglobulins antibody response, complement factors 3 and 4 , acute phase reactants and interferonproducing capacity of blood lymphocytes. Clin Exp Immunol 1977;30:50-5.

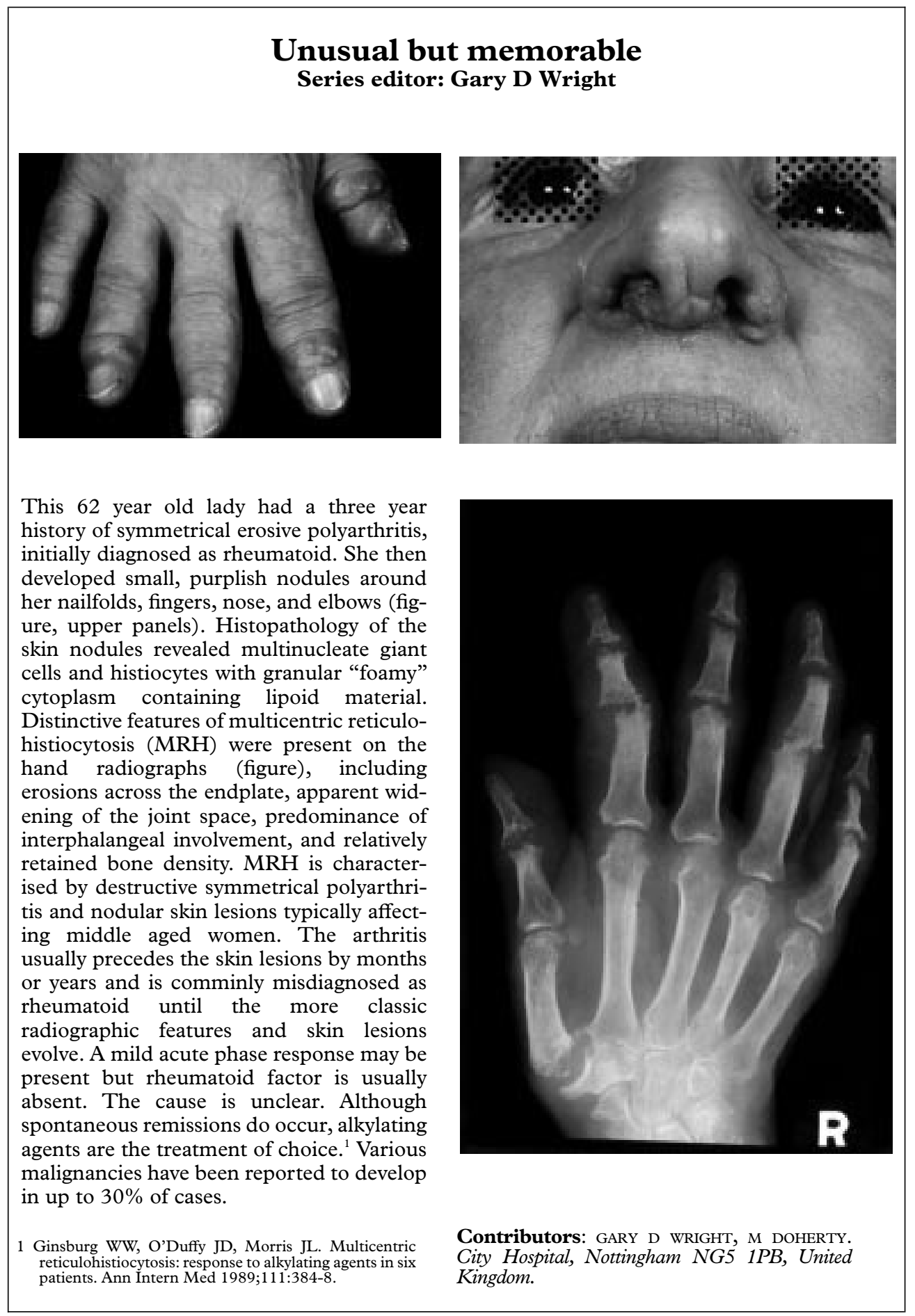

